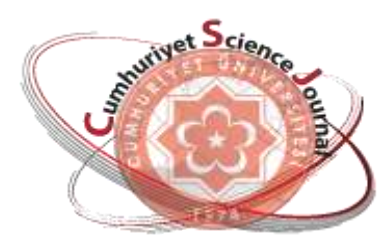

e-ISSN: 2587-246X

ISSN: 2587-2680

\section{Cuculaniyet Seionee Journal $\operatorname{csg}$}

Cumhuriyet Sci. J., Vol.39-3(2018) 581-596

\title{
Approximation by Double Deferred Nörlund Means of Double Fourier Series for Lipschitz Functions
}

\author{
Şeyda SEZGEK, IIhan DAĞADUR* \\ Mersin University Department of Mathematics, Mersin, TURKEY \\ http://dx.doi.org/10.17776/csj.439832
}

Received: 02.07.2018; Accepted: 24.09.2018

\begin{abstract}
In this paper, the concept of Double Deferred Nörlund means is defined and some important results are obtained. Particularly, we investigate the rate of uniform approximation by Double Deferred Nörlund means of the rectangular partial sums of the double Fourier series of a function $f(x, y)$ belong to $\operatorname{Lip}_{\alpha} 0<\alpha \leq 1$ on the two dimensional region $-\pi \leq x, y \leq \pi$. We also obtain the rate of uniform approximation by Double Deferred Cesáro means.
\end{abstract}

2010 Mathematics Subject Classifiction: 40C05, 42A25, 42A10, 44A99

Keywords: Functions of bounded variation, Ostrowski type inequalities, Riemann-Stieltjes integrals.

\section{Lipschitz Fonksiyonları için Çift İndisli Fourier Serilerinin Double Deferred Nörlund Ortalamasıyla Yaklaşım}

\begin{abstract}
Özet. Bu çalışmada, Double Deferred Nörlund ortalaması kavramı tanımlandı ve bazı önemli sonuçlar elde edildi. Özellikle, iki boyutlu $-\pi \leq x, y \leq \pi$ torus bölgesinde $\operatorname{Lip}_{\alpha} 0<\alpha \leq 1$ sinıfina ait $f(x, y)$ fonksiyonunun çift indisli Fourier serisinin dikdörtgensel kısmi toplamlarının çift indisli Deferred Nörlund ortalamasıyla düzgün yaklaşım oranını araştırıyoruz. Ayrıca; Double Deferred Cesáro ortalaması yardımı ile düzgün yaklaşım oranı elde ediyoruz.
\end{abstract}

Anahtar Kelimeler: Sınırlı varyasyonlu fonksiyon, Ostrowski tipli eşitsizlikler, Riemann-Stieltjes integralleri.

\section{INTRODUCTION}

In [3], the first study on double sequences was examined by Bromwich. And then it was investigated by many authors such as Hardy [7], Moricz [14], Tripathy [19], Başarır and Sonalcan [2]. The concept of regular convergence for double sequences was defined by Hardy [7]. Many recent improvements containing the summability by four dimensional matrices might be found in [25]. Various approaches of the Fourier series have been studied by various authors [8-11, 17, 21-23]. In [4], Deferred Cesáro mean $D_{\beta, \gamma}$ (Double Deferred Cesáro mean) for a double sequence $x=\left(x_{n m}\right)$ is defined and several theorems on this subject are given. Let $\left\{p_{j k}: j, k=0,1, \ldots\right\}$ be a double sequence of nonnegative numbers $p_{00}>0$ . Its partial sum is defined as

$P_{n m}=\sum_{j=0}^{n} \sum_{k=0}^{m} p_{j k} \quad(m, n=0,1, \ldots)$. 
Let $\left\{s_{j k}: j, k=0,1, \ldots\right\}$ be a double sequence of complex numbers. The Nörlund means $N_{n m}$ are defined by

$$
N_{n m}=\sum_{j=0}^{n} \sum_{k=0}^{m} p_{n-j, m-k} s_{j k}
$$

Let $f(x, y)$ be a complex valued function of period $2 \pi$ with respect to each of the variables, and integrable defined on the two dimensional real torus $\mathcal{Q}:=\left\{(x, y) \in \mathbb{R}^{2}:-\pi<x \leq \pi,-\pi<y \leq \pi\right\}$, i.e., $f \in L^{1}(Q)$. We consider the double Fourier series of $f$ defined by

$$
f(x, y) \approx \sum_{j=-\infty}^{\infty} \sum_{k=-\infty}^{\infty} c_{j k} e^{i(j x+k y)}
$$

where

$$
c_{j k}=\frac{1}{(2 \pi)^{2}} \int_{-\pi}^{\pi} \int_{-\pi}^{\pi} f(u, v) e^{-i(j u+k v)} d u d v \quad(j, k=\ldots,-1,0,1, \ldots) .
$$

We write the double sequence of (symmetric) rectangular partial sums for the series (1.1) as follows

$$
s_{n m}(x, y)=\sum_{j=-n}^{n} \sum_{k=-m}^{m} c_{j k} e^{i(j x+k y)} \quad(m, n=0,1, \ldots) .
$$

We say that the function $f$ belongs to Lipschitz class of order $\alpha$ for some $\alpha>0$, if

$$
\begin{array}{rlr}
w(\delta ; f) & =\sup _{(x, y) \in Q} \sup _{\left\{u^{2}+v^{2}\right\}^{1 / 2} \leq \delta}|f(x+u, y+v)-f(x, y)| \\
& \leq C \delta^{\alpha} \quad(\delta>0)
\end{array}
$$

where the constant $C$ does not depend on $\delta$. The quantity $w(\delta ; f)$ is called the (total) modulus of continuity of the function $f$.

Clearly, if $f \in \operatorname{Lip} \alpha$ for some $\alpha>0$, then $f$ is necessarily continuous everywhere. Only the case $0<\alpha \leq 1$ is interesting. If $\alpha>1$, then $\partial f / \partial x$ and $\partial f / \partial y$ exist and are zero everywhere, so $f$ must be a constant.

Condition (1.2) can be rewritten as

$$
|f(x+u, y+v)-f(x, y)| \leq C\left\{u^{2}+v^{2}\right\}^{\alpha / 2}
$$

for every real $x, y, u, v$; or equivalently,

$$
|f(x+u, y+v)-f(x, y)| \leq C\left(|u|^{\alpha}+|v|^{\alpha}\right) .
$$

From above inequalities yield

$$
|\phi(u, v)| \leq C\left(|u|^{\alpha}+|v|^{\alpha}\right) .
$$

We will use some well-known estimates. 
$D_{j}(u)$ is the Dirichlet kernels in terms of $u$

$$
D_{j}(u)=\frac{1}{2}+\sum_{\sigma=1}^{j} \cos \sigma u=\frac{\sin \left(j+\frac{1}{2}\right) u}{2 \sin \frac{1}{2} u}
$$

For $j=0,1, \ldots$,

$$
\left|D_{j}(u)\right|<j+1 \quad \text { for every } u \text {. }
$$

For $a, b=0,1, \ldots ; a \leq b$,

$$
\sum_{j=a}^{b} \sin \left(j+\frac{1}{2}\right) u=\frac{\cos a u-\cos (b+1) u}{2 \sin \frac{1}{2} u} .
$$

By the following inequalities

$$
\frac{\sin u}{u} \geq \frac{2}{\pi} \quad 0<u \leq \frac{\pi}{2}
$$

we have

$$
\left|\sum_{j=a}^{b} \sin \left(j+\frac{1}{2}\right) u\right| \leq \frac{\pi}{u} \quad \text { for } 0<u \leq \pi
$$

and we obtain

$$
\left|\sum_{j=a}^{b} D_{j}(u)\right|=\frac{\cos a u-\cos (b+1) u}{\left(2 \sin \frac{1}{2} u\right)^{2}} \leq \frac{\pi^{2}}{2 u^{2}} \quad \text { for } 0<u \leq \pi .
$$

\section{MAIN RESULTS}

Motivating by Moricz [13] we make the following definition.

Let $\left(a_{n}\right),\left(b_{n}\right),\left(t_{m}\right)$ and $\left(r_{m}\right)$ are sequences of nonnegative integers satisfying the conditions $a_{n}<b_{n}, t_{m}<r_{m}$ and $\lim _{n \rightarrow \infty} b_{n}=\infty, \lim _{m \rightarrow \infty} r_{m}=\infty$. Let $\left\{p_{j k}: j, k=0,1, \ldots\right\}$ be a double sequence of nonnegative numbers such that $p_{00}>0$. Then $P_{n m}^{\beta \gamma}$ is defined as follows,

$$
P_{n m}^{\beta \gamma}=\sum_{j=a_{n}+1}^{b_{n}} \sum_{k=t_{m}+1}^{r_{m}} p_{j k} \quad(n, m=0,1, \ldots)
$$

where $\beta(n)=\left(a_{n}, b_{n}\right)$ and $\gamma(m)=\left(t_{m}, r_{m}\right)$.

Now, we have the following definition

Definition 2.1. Let $\left\{s_{j k}: j, k=0,1, \ldots\right\}$ be a double sequence of complex numbers; Double Deferred Nörlund means $D_{\beta}^{\gamma} N_{n m}$ is defined as follows

$$
D_{\beta}^{\gamma} N_{n m}=\frac{1}{P_{n m}^{\beta \gamma}} \sum_{j=a_{n}+1}^{b_{n}} \sum_{k=t_{m}+1}^{r_{m}} p_{b_{n}-j, r_{m}-k} s_{j k} .
$$


It is clear that $D_{\beta}^{\gamma} N_{n m}$ method is regular.

The $D_{\beta}^{\gamma} N_{n m}$ method for the double sequence $\left\{s_{n m}(x, y)\right\}$ are defined as

$$
D_{\beta}^{\gamma} N_{n m}(x, y)=\frac{1}{P_{n m}^{\beta \gamma}} \sum_{j=a_{n}+1}^{b_{n}} \sum_{k=t_{m}+1}^{r_{m}} p_{b_{n}-j, r_{m}-k} s_{j k}(x, y) \quad(n, m=0,1, \ldots) .
$$

The following representation is important, for $n, m=0,1, \ldots$

$$
D_{\beta}^{\gamma} N_{n m}(x, y)=\frac{1}{\pi^{2}} \int_{-\pi}^{\pi} \int_{-\pi}^{\pi} f(x+u, y+v) D_{\beta}^{\gamma} K_{n m}(u, v) d u d v
$$

where $D_{\beta}^{\gamma} N_{n m}$-kernel of $D_{\beta}^{\gamma} K_{n m}(u, v)$ is defined by

$$
D_{\beta}^{\gamma} K_{n m}(u, v)=\frac{1}{P_{n m}^{\beta \gamma}} \sum_{j=a_{n}+1}^{b_{n}} \sum_{k=t_{m}+1}^{r_{m}} p_{b_{n}-j, r_{m}-k} D_{j}(u) D_{k}(v) \quad(n, m=0,1, \ldots) .
$$

From (2.1), we have

$$
D_{\beta}^{\gamma} N_{n m}(x, y)-f(x, y)=\frac{4}{\pi^{2}} \int_{0}^{\pi} \int_{0}^{\pi} \phi(u, v) D_{\beta}^{\gamma} K_{n m}(u, v) d u d v
$$

where

$$
\phi(u, v)=\frac{1}{4}\{f(x+u, y+v)+f(x-u, y+v)+f(x+u, y-v)+f(x-u, y-v)-4 f(x, y)\} .
$$

We will use the notations for $j, k=0,1, \ldots$,

$$
\begin{aligned}
& \Delta_{10} p_{j k}=p_{j k}-p_{j+1, k}, \\
& \Delta_{01} p_{j k}=p_{j k}-p_{j, k+1},
\end{aligned}
$$

and

$$
\Delta_{11} p_{j k}=p_{j k}-p_{j+1, k}-p_{j, k+1}+p_{j+1, k+1} .
$$

The double sequence $\left\{p_{j k}\right\}$ is nondecreasing if $\Delta_{10} p_{j k} \leq 0$ and $\Delta_{01} p_{j k} \leq 0$, and is nonincreasing if $\Delta_{10} p_{j k} \geq 0$ and $\Delta_{01} p_{j k} \geq 0$ for every $j, k=0,1, \ldots$ We also set, for $n, m=0,1, \ldots$

$$
D_{\beta}^{\gamma} q_{n m}=\frac{1}{P_{n m}^{\beta \gamma}} \sum_{k=t_{m}+1}^{r_{m}} p_{b_{n} k}, \quad D_{\beta}^{\gamma} r_{n m}=\frac{1}{P_{n m}^{\beta \gamma}} \sum_{j=a_{n}+1}^{b_{n}} p_{j r_{m}} .
$$

Let us consider the case where $\left\{p_{j k}\right\}$ is nondecreasing. Then 


$$
\begin{aligned}
\left(b_{n}-a_{n}\right) D_{\beta}^{\gamma} q_{n m} & =\frac{\left(b_{n}-a_{n}\right)}{P_{n m}^{\beta \gamma}} \sum_{k=t_{m}+1}^{r_{m}} p_{b_{n} k} \\
& \geq \frac{1}{P_{n m}^{\beta \gamma}} \sum_{j=a_{n}+1}^{b_{n}} \sum_{k=t_{m}+1}^{r_{m}} p_{j k}=1
\end{aligned}
$$

and similarly,

$$
\left(r_{m}-t_{m}\right) D_{\beta}^{\gamma} r_{n m} \geq 1
$$

We also have

$$
P_{n m}^{\beta \gamma} \leq\left(b_{n}-a_{n}\right)\left(r_{m}-t_{m}\right) p_{b_{n} r_{m}} \quad(n, m=0,1, \ldots) .
$$

In the sequel, we need the opposite inequality:

$$
\frac{\left(b_{n}-a_{n}\right)\left(r_{m}-t_{m}\right) p_{b_{n} r_{m}}}{P_{n m}^{\beta \gamma}}=O(1) .
$$

The opposite inequality is proved for the following sequence defined below,

$$
p_{j k}=\left(b_{n}-j+1\right)^{\sigma}\left(r_{m}-k+1\right)^{\rho} \quad(\sigma, \rho \geq 0) .
$$

Condition (2.5) implies that

$$
\begin{aligned}
\left(b_{n}-a_{n}\right) D_{\beta}^{\gamma} q_{n m} & =\frac{\left(b_{n}-a_{n}\right)}{P_{n m}^{\beta \gamma}} \sum_{k=t_{m}+1}^{r_{m}} p_{b_{n} k} \\
& \leq \frac{\left(b_{n}-a_{n}\right)\left(r_{m}-t_{m}\right)}{P_{n m}^{\beta \gamma}} p_{b_{n} r_{m}}=O(1)
\end{aligned}
$$

and

$$
\left(r_{m}-t_{m}\right) D_{\beta}^{\gamma} r_{n m}=O(1)
$$

In particular, the conditions of regularity are satisfied:

$$
\lim _{n, m \rightarrow \infty} D_{\beta}^{\gamma} q_{n m}=\lim _{n, m \rightarrow \infty} D_{\beta}^{\gamma} r_{n m}=0 .
$$

Thus, we may assume that

$$
D_{\beta}^{\gamma} q_{n m}<\pi \quad \text { and } \quad D_{\beta}^{\gamma} r_{n m}<\pi \quad(n, m=0,1, \ldots) .
$$

If $\left\{p_{j k}\right\}$ is nonincreasing, then

$$
\left(b_{n}-a_{n}\right) D_{\beta}^{\gamma} q_{n m} \leq 1 \quad \text { and } \quad\left(r_{m}-t_{m}\right) D_{\beta}^{\gamma} r_{n m} \leq 1 .
$$

In the special case where

$$
\lim _{n, m \rightarrow \infty} p_{n m}>0
$$


we have

$$
\frac{1}{\left(b_{n}-a_{n}\right) D_{\beta}^{\gamma} q_{n m}} \leq \frac{p_{a_{n}+1, t_{m}+1}}{p_{b_{n} r_{m}}}=O(1) \quad \text { and } \quad \frac{1}{\left(r_{m}-t_{m}\right) D_{\beta}^{\gamma} r_{n m}} \leq \frac{p_{a_{n}+1, t_{m}+1}}{p_{b_{n} r_{m}}}=O(1)
$$

The following result is analogue of a result of Moore [12, page 39] which follows almost the same lines, so the details are omitted.

Theorem 2.2. If $\left\{p_{j k}: j, k=0,1, \ldots, p_{00}>0\right\}$, then the necessary and sufficient conditions for the regularity of the $D_{\beta}^{\gamma} N_{n m}$-method of summability are

$$
\lim _{n, m \rightarrow \infty} \frac{1}{P_{n m}^{\beta \gamma}} \sum_{k=t_{m}+1}^{r_{m}} p_{b_{n}-j, k}=0 \quad\left(j=0,1, \ldots ; b_{n} \geq j\right)
$$

and

$$
\lim _{n, m \rightarrow \infty} \frac{1}{P_{n m}^{\beta \gamma}} \sum_{j=a_{n}+1}^{b_{n}} p_{j, r_{m}-k}=0 \quad\left(k=0,1, \ldots ; r_{m} \geq k\right) .
$$

Lemma 2.3. Let $\left\{p_{j k}>0: j, k=0,1, \ldots\right\}$ be a nondecreasing double sequence such that $\Delta_{11} p_{j k}$ is of fixed sign. Then

$$
\begin{array}{rlrl}
\left|D_{\beta}^{\gamma} K_{n m}(u, v)\right| & \leq\left(b_{n}+1\right)+\left(r_{m}+1\right), & \forall u, v \\
& \leq \frac{\pi^{2}}{2 u^{2} P_{n m}^{\beta \gamma}} \sum_{k=t_{m}+1}^{r_{m}}(k+1)\left[p_{b_{n}-a_{n}-1, r_{m}-k-1}-p_{0, r_{m}-k}\right], & & \forall v \text { and } 0<u \leq \pi \\
& \leq \frac{\pi^{2}}{2 v^{2} P_{n m}^{\beta \gamma}} \sum_{j=a_{n}+1}^{b_{n}}(j+1)\left[p_{b_{n}-j-1, r_{m}-t_{m}-1}-p_{b_{n}-j, 0}\right], & & \forall u \text { and } 0<v \leq \pi \\
& \leq \frac{5 \pi^{4} p_{b_{n} r_{m}}}{4 u^{2} v^{2} P_{n m}^{\beta \gamma}}, & 0<u, v \leq \pi .
\end{array}
$$

Proof. From (1.4),

which is (2.11).

$$
\begin{aligned}
\left|D_{\beta}^{\gamma} K_{n m}(u, v)\right| & \leq \frac{1}{P_{n m}^{\beta \gamma}} \sum_{j=a_{n}+1}^{b_{n}} \sum_{k=t_{m}+1}^{r_{m}} p_{b_{n}-j, r_{m}-k}\left|D_{j}(u)\right|\left|D_{k}(v)\right| \\
& \leq \frac{1}{P_{n m}^{\beta \gamma}} \sum_{j=a_{n}+1}^{b_{n}} \sum_{k=t_{m}+1}^{r_{m}}(j+1)(k+1) p_{b_{n}-j, r_{m}-k} \\
& \leq \frac{\left(b_{n}+1\right)\left(r_{m}+1\right)}{P_{n m}^{\beta \gamma}} \sum_{j=a_{n}+1}^{b_{n}} \sum_{k=t_{m}+1}^{r_{m}} p_{j k} \\
& =\left(b_{n}+1\right)\left(r_{m}+1\right)
\end{aligned}
$$

Again from (1.4), we have 


$$
\begin{aligned}
P_{n m}^{\beta \gamma}\left|D_{\beta}^{\gamma} K_{n m}(u, v)\right| & \leq \sum_{k=t_{m}+1}^{r_{m}}\left|\sum_{j=a_{n}+1}^{b_{n}} p_{b_{n}-j, r_{m}-k} D_{j}(u)\right|\left|D_{k}(v)\right| \\
& \leq \sum_{k=t_{m}+1}^{r_{m}}(k+1)\left|\sum_{j=a_{n}+1}^{b_{n}} p_{b_{n}-j, r_{m}-k} D_{j}(u)\right|
\end{aligned}
$$

If we apply the Abel's transformation to the inner sum, for each $k$

$$
\begin{aligned}
\sum_{j=a_{n}+1}^{b_{n}} p_{b_{n}-j, r_{m}-k} D_{j}(u)= & \left(\sum_{j=0}^{b_{n}}-\sum_{k=0}^{r_{m}}\right) p_{b_{n}-j, r_{m}-k} D_{j}(u)=-\sum_{j=1}^{b_{n}} \Delta_{10} p_{b_{n}-j, r_{m}-k} \sum_{l=0}^{j-1} D_{l}(u) \\
& +p_{0, r_{m}-k} \sum_{l=0}^{b_{n}} D_{l}(u)+\sum_{j=1}^{a_{n}} \Delta_{10} p_{b_{n}-j, r_{m}-k} \sum_{l=0}^{j-1} D_{l}(u)+p_{0, r_{m}-k} \sum_{l=0}^{a_{n}} D_{l}(u) .
\end{aligned}
$$

Since we consider $\left\{p_{j k}\right\}$ as nondecreasing in $j$ and (1.7), we obtain

$$
\begin{aligned}
\left|\sum_{j=a_{n}+1}^{b_{n}} p_{b_{n}-j, r_{m}-k} D_{j}(u)\right| & =\frac{\pi^{2}}{2 u^{2}}\left(\sum_{j=1}^{a_{n}} \Delta_{10} p_{b_{n}-j, r_{m}-k}-p_{0, r_{m}-k}+p_{0, r_{m}-k}-\sum_{j=1}^{b_{n}} \Delta_{10} p_{b_{n}-j, r_{m}-k}\right) \\
& \leq \frac{\pi^{2}}{2 u^{2}} \sum_{j=a_{n}+1}^{b_{n}} \Delta_{10} p_{b_{n}-j, r_{m}-k}=\frac{\pi^{2}}{2 u^{2}}\left[p_{b_{n}-a_{n}-1, r_{m}-k}-p_{0, r_{m}-k}\right] .
\end{aligned}
$$

If we write (2.15) again, we have

$$
\left|D_{\beta}^{\gamma} K_{n m}(u, v)\right| \leq \frac{\pi^{2}}{2 P_{n m}^{\beta \gamma} u^{2}} \sum_{k=t_{m}+1}^{r_{m}}(k+1)\left[p_{b_{n}-a_{n}-1, r_{m}-k}-p_{0, r_{m}-k}\right] .
$$

(2.13) can be shown similarly. We perform the double Abel's transformation to prove (2.14)

$$
\begin{aligned}
P_{n m}^{\beta \gamma} D_{\beta}^{\gamma} K_{n m}(u, v) & =\sum_{j=a_{n}+1}^{b_{n}} \sum_{k=t_{m}+1}^{r_{m}} p_{b_{n}-j, r_{m}-k} D_{j}(u) D_{k}(v) \\
= & \sum_{j=a_{n}+1}^{b_{n}-1} \sum_{k=t_{m}+1}^{r_{m}-1} \Delta_{11} p_{b_{n}-j, r_{m}-k} \sum_{i=0}^{j-1} D_{i}(u) \sum_{l=0}^{k-1} D_{l}(v)+\sum_{j=a_{n}+1}^{b_{n}-1} \Delta_{10} p_{b_{n}-j, 0} \sum_{i=0}^{j-1} D_{i}(u) \sum_{l=0}^{r_{m}} D_{l}(v) \\
& -\sum_{j=a_{n}+1}^{b_{n}-1} \Delta_{10} p_{b_{n}-j, r_{m}-t_{m}-1} \sum_{i=0}^{j-1} D_{i}(u) \sum_{l=0}^{t_{m}} D_{l}(v)+\sum_{k=t_{m}+1}^{r_{m}-1} \Delta_{01} p_{0, r_{m}-k} \sum_{i=0}^{b_{n}} D_{i}(u) \sum_{l=0}^{k-1} D_{l}(v) \\
& -\sum_{k=t_{m}+1}^{r_{m}-1} \Delta_{01} p_{b_{n}-a_{n}-1, r_{m}-k} \sum_{i=0}^{t_{n}} D_{i}(u) \sum_{l=0}^{k-1} D_{l}(v)+p_{b_{n} r_{m}} \sum_{i=0}^{b_{n}} D_{i}(u) \sum_{l=0}^{r_{m}} D_{l}(v) \\
& -p_{b_{n}-a_{n}-1, r_{m}} \sum_{i=0}^{a_{n}} D_{i}(u) \sum_{l=0}^{r_{m}} D_{l}(v)-p_{b_{n}, r_{m}-t_{m}-1} \sum_{i=0}^{b_{n}} D_{i}(u) \sum_{l=0}^{t_{m}} D_{l}(v) \\
& +p_{b_{n}-a_{n}-1, r_{m}-t_{m}-1} \sum_{i=0}^{a_{n}} D_{i}(u) \sum_{l=0}^{t_{m}} D_{l}(v),
\end{aligned}
$$

from (1.7) 


$$
\begin{aligned}
P_{n m}^{\beta \gamma}\left|D_{\beta}^{\gamma} K_{n m}(u, v)\right| \leq & \frac{\pi^{4}}{4 u^{2} v^{2}}\left(\sum_{j=a_{n}+1}^{b_{n}} \sum_{k=t_{m}+1}^{r_{m}}\left|\Delta_{11} p_{b_{n}-j, r_{m}-k}\right|+\sum_{j=a_{n}+1}^{b_{n}} \Delta_{10} p_{b_{n}-j, 0}\right. \\
& -\sum_{j=a_{n}+1}^{b_{n}} \Delta_{10} p_{b_{n}-j, r_{m}-t_{m}-1}+\sum_{k=t_{m}+1}^{r_{m}} \Delta_{01} p_{0, r_{m}-k}-\sum_{k=t_{m}+1}^{r_{m}} \Delta_{01} p_{b_{n}-a_{n}-1, r_{m}-k} \\
& \left.+p_{b_{n} r_{m}}-p_{b_{n}, r_{m}-t_{m}-1}-p_{b_{n}-a_{n}-1, r_{m}-t_{m}-1}-p_{b_{n}-a_{n}-1, r_{m}}\right) .
\end{aligned}
$$

Since $\Delta_{11} p_{j k}$ is fixed, we get

$$
\sum_{j=a_{n}+1}^{b_{n}} \sum_{k=t_{m}+1}^{r_{m}}\left|\Delta_{11} p_{b_{n}-j, r_{m}-k}\right|=\left|p_{b_{n}-a_{n}, r_{m}-t_{m}}-p_{b_{n}-a_{n}, 0}-p_{0, r_{m}-t_{m}}+p_{00}\right| .
$$

According to (2.18), if $\Delta_{11} p_{j k} \geq 0$, we obtained

$$
\begin{aligned}
P_{n m}^{\beta \gamma}\left|D_{\beta}^{\gamma} K_{n m}(u, v)\right| & \leq \frac{\pi^{4}}{4 u^{2} v^{2}}\left(p_{b_{n}-a_{n}, r_{m}-t_{m}}+p_{b_{n}, r_{m}-t_{m}-1}+p_{b_{n}-a_{n}-1, r_{m}}+p_{b_{n}-a_{n}-1, r_{m}-t_{m}-1}+p_{b_{n}, r_{m}}\right) \\
& \leq \frac{5 \pi^{4}}{4 u^{2} v^{2}} p_{b_{n}, r_{m}}
\end{aligned}
$$

and if $\Delta_{11} p_{j k} \leq 0$, we obtained

$$
P_{n m}^{\beta \gamma}\left|D_{\beta}^{\gamma} K_{n m}(u, v)\right| \leq \frac{3 \pi^{4}}{4 u^{2} v^{2}} p_{b_{n} r_{m}} .
$$

Lemma 2.4. Let $\left\{p_{j k}>0: j, k=0,1 \ldots\right\}$ be a nonincreasing double sequence such that $\Delta_{11} p_{j k}$ is fixed and let $p=[1 / u], \eta=[1 / v]$ where $[$.$] means the integral part. Then$

$$
\begin{aligned}
\left|D_{\beta}^{\gamma} K_{n m}(u, v)\right| & \leq\left(b_{n}+1\right)\left(r_{m}+1\right) & \forall u, v \\
& \leq \frac{\pi(\pi+1)}{2} \frac{1}{P_{n m}^{\beta \gamma} u} \sum_{k=t_{m}+1}^{r_{m}}(k+1) \sum_{j=0}^{p} p_{j, r_{m}-k}, & \forall v \text { and } 0<u \leq \pi \\
& \leq \frac{\pi(\pi+1)}{2} \frac{1}{P_{n m}^{\beta \gamma} v} \sum_{j=a_{n}+1}^{b_{n}}(j+1) \sum_{k=0}^{\eta} p_{b_{n}-j, k}, & \forall u \text { and } 0<v \leq \pi \\
& \leq \frac{\pi^{2}\left(1+2 \pi+3 \pi^{2}\right)}{4} \frac{p_{p \eta}}{P_{n m}^{\beta \gamma} u v} &
\end{aligned}
$$

Proof. Equation (2.19) coincides with (2.11) as we remarked in the proof of Lemma 2.3.

From (2.15) and (1.5),

$$
\begin{aligned}
p_{n m}^{\beta \gamma}\left|D_{\beta}^{\gamma} K_{n m}(u, v)\right| & \leq \sum_{k=t_{m}+1}^{r_{m}}(k+1)\left|\sum_{j=a_{n}+1}^{b_{n}} p_{b_{n}-j, r_{m}-k} D_{j}(u)\right| \\
& \leq \frac{\pi}{2 u} \sum_{k=t_{m}+1}^{r_{m}}(k+1)\left|\sum_{j=0}^{b_{n}-a_{n}-1} p_{b_{n}-j, r_{m}-k} \sin \left(b_{n}-j+\frac{1}{2}\right) u\right| .
\end{aligned}
$$


By a simple estimate the inner sum can be written as follows for each $\mathrm{k}$,

$$
\begin{aligned}
& \left|\sum_{j=0}^{b_{n}-a_{n}-1} p_{b_{n}-j, r_{m}-k} \sin \left(b_{n}-j+\frac{1}{2}\right) u\right| \\
& \leq \sum_{j=0}^{p} p_{b_{n}-j, r_{m}-k}+\left|\sum_{j=p+1}^{b_{n}-a_{n}-1} p_{j, r_{m}-k} \sin \left(b_{n}-j+\frac{1}{2}\right) u\right| .
\end{aligned}
$$

From the Abel's transformation, we have

$$
\begin{aligned}
\sum_{j=p+1}^{b_{n}-a_{n}-1} p_{j, r_{m}-k} \sin \left(b_{n}-j+\frac{1}{2}\right) u= & \sum_{j=p+1}^{b_{n}-a_{n}-2} \Delta_{10} p_{j-r_{m}-k} \sum_{l=p+1}^{j} \sin \left(b_{n}-j+\frac{1}{2}\right) u \\
& +p_{b_{n}-a_{n}-1, r_{m}-k} \sum_{l=p+1}^{b_{n}} \sin \left(b_{n}-j+\frac{1}{2}\right) u .
\end{aligned}
$$

From (1.6), $\left\{p_{j k}\right\}$ is nonincreasing in $j$ and $\frac{1}{u}<\rho+1$ we have

$$
\left|\sum_{j=p+1}^{b_{n}-a_{n}-1} p_{b_{n}-j, r_{m}-k} \sin \left(b_{n}-j+\frac{1}{2}\right) u\right| \leq \frac{\pi}{u} p_{\rho+1, r_{m}-k} \leq \pi(\rho+1) p_{\rho+1, r_{m}-k} \leq \pi \sum_{j=0}^{\rho} p_{j, r_{m}-k}
$$

Combining (2.18), (2.24) and (2.26) yield (2.20).

(2.21) can be proved similarly. Now, let's prove (2.22) beginning the following inequality,

$$
\begin{aligned}
P_{n m}^{\beta \gamma}\left|D_{\beta}^{\gamma} K_{n m}(u, v)\right| & =\left|\sum_{j=a_{n}+1}^{b_{n}} \sum_{k=t_{m}+1}^{r_{m}} p_{b_{n}-j, r_{m}-k} D_{j}(u) D_{k}(v)\right| \\
& =\left|\sum_{j=0}^{b_{n}-a_{n}-1} \sum_{k=0}^{r_{m}-t_{m}-1} p_{j k} D_{b_{n}-j}(u) D_{r_{m}-k}(v)\right| \\
& \leq \frac{\pi^{2}}{4 u v}\left|\sum_{j=0}^{b_{n}-a_{n}-1} \sum_{k=0}^{b_{n}-a_{n}-1} p_{j k} \sin \left(b_{n}-j+\frac{1}{2}\right) u \sin \left(r_{m}-k+\frac{1}{2}\right) v\right| .
\end{aligned}
$$

We divide the double sum into four parts:

$$
\begin{aligned}
& \left|\sum_{j=0}^{\mid b_{n}-a_{n}-1 b_{n}-a_{n}-1} \sum_{k=0}^{n} p_{j k} \sin \left(b_{n}-j+\frac{1}{2}\right) u \sin \left(r_{m}-k+\frac{1}{2}\right) v\right| \leq \sum_{j=0}^{\rho} \sum_{k=0}^{\eta} p_{j k}+\sum_{k=0}^{\eta}\left|\sum_{j=\rho+1}^{b_{n}-a_{n}-1} p_{j k} \sin \left(b_{n}-j+\frac{1}{2}\right) u\right| \\
& \quad+\sum_{j=0}^{\rho}\left|\sum_{k=\eta+1}^{r_{m}-t_{m}-1} p_{j k} \sin \left(r_{m}-k+\frac{1}{2}\right) v\right|+\left|\sum_{j=\rho+1}^{b_{n}-a_{n}-1} \sum_{k=\eta+1}^{r_{m}-t_{m}-1} p_{j k} \sin \left(b_{n}-j+\frac{1}{2}\right) u \sin \left(r_{m}-k+\frac{1}{2}\right) v\right| \\
& =P_{\rho \eta}+A_{1}+A_{2}+A_{3} .
\end{aligned}
$$

For $A_{1}$ we can perform the Abel's transformation similar to (2.25) and conclude that 
Thus we have the following inequation

$$
\begin{aligned}
\sum_{j=p+1}^{b_{n}-a_{n}-1} p_{b_{n}-j, r_{m}-k} \sin \left(b_{n}-j+\frac{1}{2}\right) u & \leq \sum_{j=\rho+1}^{b_{n}-a_{n}-2} \Delta_{10} p_{j k}\left|\sum_{l=p+1}^{j} \sin \left(b_{n}-l+\frac{1}{2}\right) u\right| \\
& +p_{b_{n}-a_{n}-1, k}\left|\sum_{l=p+1}^{b_{n}-a_{n}-1} \sin \left(b_{n}-l+\frac{1}{2}\right) u\right| \\
\leq & \frac{\pi}{u} p_{\rho+1, k} \leq \pi(\rho+1) p_{\rho+1, k} \leq \pi \sum_{j=0}^{\rho} p_{j k}
\end{aligned}
$$

$$
A_{1} \leq \pi P_{\rho \eta}
$$

So similarly,

$$
A_{2} \leq \pi P_{\rho \eta}
$$

For A3, we perform the double Abel's transformation:

$$
\begin{aligned}
& \sum_{j=\rho+1}^{b_{n}-a_{n}-1} \sum_{k=\eta+1}^{r_{m}-t_{m}-1} p_{j k} \sin \left(b_{n}-j+\frac{1}{2}\right) u \sin \left(r_{m}-k+\frac{1}{2}\right) v \\
& =\sum_{j=\rho+1}^{b_{n}-a_{n}-2 r_{m}-t_{m}-2} \sum_{k=\eta+1}^{r_{n}} \Delta_{11} p_{j k} \sum_{i=\rho+1}^{j} \sin \left(b_{n}-i+\frac{1}{2}\right) u \sum_{l=\eta+1}^{k} \sin \left(r_{m}-l+\frac{1}{2}\right) v \\
& \quad+\sum_{j=\rho+1}^{b_{n}-a_{n}-2} \Delta_{10} p_{j r_{m}} \sum_{i=\rho+1}^{j} \sin \left(b_{n}-i+\frac{1}{2}\right) u \sum_{l=\eta+1}^{r_{m}-t_{m}-2} \sin \left(r_{m}-l+\frac{1}{2}\right) v \\
& +\sum_{k=\eta+1}^{r_{m}-t_{m}-2} \Delta_{01} p_{j k} \sum_{i=\rho+1}^{b_{n}-a_{n}-2} \sin \left(b_{n}-i+\frac{1}{2}\right) u \sum_{l=\eta+1}^{k} \sin \left(r_{m}-l+\frac{1}{2}\right) v \\
& \quad+p_{b_{n} r_{m}} \sum_{i=\rho+1}^{b_{n}-a_{n}-1} \sin \left(b_{n}-i+\frac{1}{2}\right) u \sum_{l=\eta+1}^{r_{m}-t_{m}-1} \sin \left(r_{m}-l+\frac{1}{2}\right) v .
\end{aligned}
$$

By (1.6),

$$
\begin{aligned}
& \left|\sum_{j=\rho+1}^{b_{n}-a_{n}-1} \sum_{k=\eta+1}^{r_{m}-t_{m}-1} p_{j k} \sin \left(b_{n}-j+\frac{1}{2}\right) u \sin \left(r_{m}-k+\frac{1}{2}\right) v\right| \\
& \leq \frac{\pi^{2}}{u v}\left\{\left|\sum_{j=\rho+1}^{b_{n}-a_{n}-1} \sum_{k=\eta+1}^{r_{m}-t_{m}-1} \Delta_{11} p_{j k}\right|+\sum_{j=\rho+1}^{b_{n}-a_{n}-2} \Delta_{10} p_{j r_{m}}+\sum_{k=\eta+1}^{r_{m}-t_{m}-2} \Delta_{01} p_{b_{n} k}+p_{b_{n} r_{m}}\right\} \\
& =\frac{\pi^{2}}{u v} p_{\rho+1, \eta+1} \quad\left(\Delta_{11} p_{j k} \geq 0\right) \\
& =\frac{\pi^{2}}{u v}\left(-2 p_{b_{n} r_{m}}+2 p_{\rho+1, r_{m}}+2 p_{b_{n}, \eta+1}-p_{\rho+1, \eta+1}\right) \leq \frac{3 \pi^{2}}{u v} p_{\rho+1, \eta+1} \quad\left(\Delta_{11} p_{j k} \leq 0\right) .
\end{aligned}
$$

Hence, the following inequality is obtained

$$
A_{3} \leq \frac{3 \pi^{2}}{u v} p_{\rho+1, \eta+1} \leq 3 \pi^{2}(\rho+1)(\eta+1) p_{\rho+1, \eta+1} \leq 3 \pi^{2} \sum_{j=0}^{\rho} \sum_{k=0}^{\eta} p_{j k}=3 \pi^{2} P_{\rho \eta} .
$$


If we consider (2.28)-(2.31) together, we obtain

$$
\left|\sum_{j=0}^{b_{n}-a_{n}-1} \sum_{k=0}^{r_{m}-t_{m}-1} p_{j k} \sin \left(b_{n}-j+\frac{1}{2}\right) u \sin \left(r_{m}-k+\frac{1}{2}\right) v\right| \leq\left(1+2 \pi+3 \pi^{2}\right) P_{\rho \eta} .
$$

Thus (2.27) implies (2.22).

Theorem 2.5. Let $\left\{p_{j k}>0: j, k=0,1 \ldots\right\}$ be a nondecreasing double sequence such that $\Delta_{11} p_{j k}$ is fixed and condition (2.5) is satisfied. If $f \in$ Lip $\alpha$ for some $0<\alpha<1$, then

$$
\begin{aligned}
& \sup _{(x, y) \in \square}\left|D_{\beta}^{\gamma} N_{n m}(x, y)-f(x, y)\right| \\
& = \begin{cases}O\left(\left(D_{\beta}^{\gamma} q_{n m}\right)^{\alpha}+\left(D_{\beta}^{\gamma} r_{n m}\right)^{\alpha}\right) & , \quad 0<\alpha<1 \\
O\left(D_{\beta}^{\gamma} q_{n m} \ln \frac{\pi}{D_{\beta}^{\gamma} q_{n m}}+D_{\beta}^{\gamma} r_{n m} \ln \frac{\pi}{D_{\beta}^{\gamma} r_{n m}}\right), & \alpha=1 .\end{cases}
\end{aligned}
$$

Proof. We start with representation (2.2). Let

$$
\begin{aligned}
& \frac{\pi^{2}}{4}\left|D_{\beta}^{\gamma} N_{n m}(x, y)-f(x, y)\right| \\
& \leq\left\{\int_{0}^{D_{\beta}^{\gamma} q_{n m}} \int_{0}^{\gamma}+\int_{D_{\beta}^{\gamma} q_{n m}}^{\pi} \int_{0}^{D_{\beta}^{\gamma} r_{n m}}+\int_{0}^{D_{\beta}^{\gamma} q_{n m}} \int_{D_{\beta}^{\gamma} r_{m m}}^{\pi}+\int_{D_{\beta}^{\gamma} q_{n m}}^{\pi} \int_{D^{\gamma} r_{m m}}^{\pi}\right\}|\phi(u, v)|\left|K_{\beta}^{\gamma}(u, v)\right| d u d v \\
& =I_{1}+I_{2}+I_{3}+I_{4} .
\end{aligned}
$$

$\phi(u, v)$ will be taken as estimated in (1.3) and the appropriate estimate of Lemma 2.3 is substituted for the kernel $D_{\beta}^{\gamma} K_{n m}(u, v)$. From (2.11), for $\alpha>0$

$$
\begin{aligned}
I_{1} & \leq\left(b_{n}+1\right)\left(r_{m}+1\right) \int_{0}^{D_{\beta}^{\gamma} q_{n m}} \int_{0}^{\gamma}\left(u^{\gamma} r_{m m}+v^{\alpha}\right) d u d v \\
& =\frac{1}{\alpha+1}\left(b_{n}+1\right)\left(r_{m}+1\right) D_{\beta}^{\gamma} q_{n m} D_{\beta}^{\gamma} r_{n m}\left(\left(D_{\beta}^{\gamma} q_{n m}\right)^{\alpha}+\left(D_{\beta}^{\gamma} r_{n m}\right)^{\alpha}\right) .
\end{aligned}
$$

By (2.6) and (2.7),

$$
I_{1}=O\left(\left(D_{\beta}^{\gamma} q_{n m}\right)^{\alpha}+\left(D_{\beta}^{\gamma} r_{n m}\right)^{\alpha}\right)
$$

By (2.12),

$$
I_{2} \leq \frac{\pi^{2}}{2 P_{n m}^{\beta \gamma}} \sum_{k=t_{m}+1}^{r_{m}}(k+1)\left[p_{b_{n}-a_{n}-1, r_{m}-k}-p_{0, r_{m}-k}\right] \int_{D_{\beta}^{\gamma} q_{n m}}^{\pi} \int_{0}^{D_{\beta}^{\gamma} r_{n m}} \frac{u^{\alpha}+v^{\alpha}}{u^{2}} d u d v
$$

for $0<\alpha<1$, 


$$
I_{2} \leq \frac{\pi^{2}}{2 P_{n m}^{\beta \gamma}} \frac{D_{\beta}^{\gamma} r_{n m}}{D_{\beta}^{\gamma} q_{n m}} \sum_{k=t_{m}+1}^{r_{m}}(k+1)\left[p_{b_{n}-a_{n}-1, r_{m}-k}-p_{0, r_{m}-k}\right]\left(\frac{\left(D_{\beta}^{\gamma} q_{n m}\right)^{\alpha}}{1-\alpha}+\frac{\left(D_{\beta}^{\gamma} q_{n m}\right)^{\alpha}}{1+\alpha}\right),
$$

for $\alpha=1$,

$$
I_{2} \leq \frac{\pi^{2}}{2 P_{n m}^{\beta \gamma}} \frac{D_{\beta}^{\gamma} r_{n m}}{D_{\beta}^{\gamma} q_{n m}} \sum_{k=t_{m}+1}^{r_{m}}(k+1)\left[p_{b_{n}-a_{n}-1, r_{m}-k}-p_{0, r_{m}-k}\right]\left(D_{\beta}^{\gamma} q_{n m} \ln \frac{\pi}{D_{\beta}^{\gamma} q_{n m}}+\frac{1}{2} D_{\beta}^{\gamma} r_{n m}\right) .
$$

With the help of (2.7)

$$
\begin{aligned}
& \frac{\pi^{2}}{2 P_{n m}^{\beta \gamma}} \frac{D_{\beta}^{\gamma} r_{n m}}{D_{\beta}^{\gamma} q_{n m}} \sum_{k=t_{m}+1}^{r_{m}}(k+1)\left[p_{b_{n}-a_{n}-1, r_{m}-k}-p_{0, r_{m}-k}\right] \\
& \leq \frac{\pi^{2}}{2 P_{n m}^{\beta \gamma}} \frac{D_{\beta}^{\gamma} r_{n m}}{D_{\beta}^{\gamma} q_{n m}} \sum_{k=t_{m}+1}^{r_{m}}(k+1) p_{b_{n}, r_{m}-k}=\left(r_{m}+1\right) \frac{\pi^{2}}{2 P_{n m}^{\beta \gamma}} \frac{D_{\beta}^{\gamma} r_{n m}}{D_{\beta}^{\gamma} q_{n m}} \sum_{k=t_{m}+1}^{r_{m}} p_{b_{n}, r_{m}-k} \\
& =\left(r_{m}+1\right) D_{\beta}^{\gamma} r_{n m}=O(1) .
\end{aligned}
$$

So,

$$
I_{2}=\left\{\begin{array}{cc}
O\left(\left(D_{\beta}^{\gamma} q_{n m}\right)^{\alpha}+\left(D_{\beta}^{\gamma} r_{n m}\right)^{\alpha}\right) & , 0<\alpha<1 \\
O\left(D_{\beta}^{\gamma} q_{n m} \ln \frac{\pi}{D_{\beta}^{\gamma} q_{n m}}+D_{\beta}^{\gamma} r_{n m}\right) & , \quad \alpha=1 .
\end{array}\right.
$$

Similarly, using (2.13),

$$
I_{3}=\left\{\begin{array}{cc}
O\left(\left(D_{\beta}^{\gamma} q_{n m}\right)^{\alpha}+\left(D_{\beta}^{\gamma} r_{n m}\right)^{\alpha}\right) & , 0<\alpha<1 \\
O\left(D_{\beta}^{\gamma} q_{n m}+D_{\beta}^{\gamma} r_{n m} \ln \frac{\pi}{D_{\beta}^{\gamma} r_{n m}}\right) & , \quad \alpha=1 .
\end{array}\right.
$$

By (2.14),

$$
I_{4} \leq \frac{5 \pi^{4}}{4} \frac{p_{b_{n} r_{m}}}{P_{n m}^{\beta \gamma}} \int_{D_{\beta}^{\gamma} q_{n m}}^{\pi} \int_{D_{\beta}^{\gamma} r_{m m}}^{\pi} \frac{u^{\alpha}+v^{\alpha}}{u^{2} v^{2}} d u d v
$$

for $\alpha=1$,

$$
\frac{5 \pi^{4}}{4(1-\alpha)} \frac{p_{b_{n} r_{m}}}{D_{\beta}^{\gamma} q_{n m} D_{\beta}^{\gamma} r_{n m} P_{n m}^{\beta \gamma}}\left(D_{\beta}^{\gamma} q_{n m} \ln \frac{\pi}{D_{\beta}^{\gamma} q_{n m}}+D_{\beta}^{\gamma} r_{n m} \ln \frac{\pi}{D_{\beta}^{\gamma} r_{n m}}\right) .
$$

From (2.3), (2.4) and (2.5),

$$
\frac{p_{b_{n} r_{m}}}{D_{\beta}^{\gamma} q_{n m} D_{\beta}^{\gamma} r_{n m} P_{n m}^{\beta \gamma}}=\frac{\left(b_{n}-a_{n}\right)\left(r_{m}-t_{m}\right) p_{b_{n} r_{m}}}{\left(b_{n}-a_{n}\right) D_{\beta}^{\gamma} q_{n m}\left(r_{m}-t_{m}\right) D_{\beta}^{\gamma} r_{n m}}=O(1)
$$


As a result,

$$
I_{4}= \begin{cases}O\left(\left(D_{\beta}^{\gamma} q_{n m}\right)^{\alpha}+\left(D_{\beta}^{\gamma} r_{n m}\right)^{\alpha}\right) & , \quad 0<\alpha<1 \\ O\left(D_{\beta}^{\gamma} q_{n m} \ln \frac{\pi}{D_{\beta}^{\gamma} q_{n m}}+D_{\beta}^{\gamma} r_{n m} \ln \frac{\pi}{D_{\beta}^{\gamma} r_{n m}}\right), & \alpha=1 .\end{cases}
$$

Collecting (2.33)-(2.37) together yields (2.32).

Theorem 2.6. Let $\left\{p_{j k}>0: j, k=0,1 \ldots\right\}$ be a nondecreasing double sequence such that $\Delta_{11} p_{j k}$ is fixed and condition (2.5) is satisfied. If $f \in$ Lip $\alpha$ for some $0<\alpha<1$, then

$$
\begin{aligned}
& \sup _{(x, y) \in \square}\left|D_{\beta}^{\gamma} N_{n m}(x, y)-f(x, y)\right| \\
& =\left\{\frac{1}{P_{n m}^{\beta \gamma}} \sum_{j=0}^{b_{n}} \sum_{k=0}^{r_{m}}\left(\frac{P_{j k}}{(j+1)^{\alpha+1}(k+1)}+\frac{P_{j k}}{(j+1)(k+1)^{\alpha+1}}\right) .\right.
\end{aligned}
$$

Proof. We use (2.33) with $D_{\beta}^{\gamma} q_{n m}$ and $D_{\beta}^{\gamma} r_{n m}$ that are replaced by $\pi /\left(b_{n}+1\right)$ and $\pi /\left(r_{m}+1\right)$, respectively. The right-hand side of (2.38) is denoted by $\Psi_{n m}$.

From (2.19), for $\alpha>0$

$$
\begin{aligned}
& I_{1} \leq\left(b_{n}+1\right)\left(r_{m}+1\right) \int_{0}^{\pi /\left(b_{n}+1\right)} \int_{0}^{\pi /\left(r_{m}+1\right)}\left(u^{\alpha}+v^{\alpha}\right) d u d v \\
& =\frac{\pi^{\alpha+2}}{\alpha+1}\left(\frac{1}{\left(b_{n}+1\right)^{\alpha}}+\frac{1}{\left(r_{m}+1\right)^{\alpha}}\right) .
\end{aligned}
$$

Since $p_{j k}$ is nonincreasing, we clearly have

$$
P_{j k} \geq\left(b_{j}+1\right)\left(r_{k}+1\right) p_{j k} \quad(j, k=0,1, \ldots) .
$$

Thus

$$
\begin{aligned}
\frac{1}{\left(b_{n}+1\right)^{\alpha}} & =\frac{1}{\left(b_{n}+1\right)^{\alpha}} \frac{1}{P_{n m}^{\beta \gamma}} \sum_{j=a_{n}+1}^{b_{n}} \sum_{k=t_{m}+1}^{r_{m}} p_{j k} \\
& \leq \frac{1}{\left(b_{n}+1\right)^{\alpha}} \frac{1}{P_{n m}^{\beta \gamma}} \sum_{j=a_{n}+1}^{b_{n}} \sum_{k=t_{m}+1}^{r_{m}} \frac{P_{j k}}{\left(b_{j}+1\right)\left(r_{k}+1\right)} \\
& \leq \frac{1}{P_{n m}^{\beta \gamma}} \sum_{j=a_{n}+1}^{b_{n}} \sum_{k=t_{m}+1}^{r_{m}} \frac{P_{j k}}{\left(b_{j}+1\right)^{\alpha+1}\left(r_{k}+1\right)}
\end{aligned}
$$

and similarly,

$$
\frac{1}{\left(r_{m}+1\right)^{\alpha}} \leq \frac{1}{P_{n m}^{\beta \gamma}} \sum_{j=a_{n}+1}^{b_{n}} \sum_{k=t_{m}+1}^{r_{m}} \frac{P_{j k}}{\left(b_{j}+1\right)\left(r_{k}+1\right)^{\alpha+1}}
$$


Combining (2.39) with the last two inequalities, we have

$$
I_{1}=O\left(\Psi_{n m}\right) .
$$

By (2.20),

$$
\begin{aligned}
I_{2} \leq & \frac{\pi(\pi+1)}{2 P_{n m}^{\beta \gamma}} \sum_{k=t_{m}+1}^{r_{m}} \int_{\pi /\left(b_{n}+1\right)}^{\pi} \int_{0}^{\pi /\left(r_{m}+1\right)} \frac{u^{\alpha}+v^{\alpha}}{u} \sum_{j=0}^{\rho} p_{j, r_{m}-k} d v d u \\
= & \frac{\pi(\pi+1)}{2 P_{n m}^{\beta \gamma}} \sum_{k=t_{m}+1}^{r_{m}}(k+1)\left\{\frac{\pi}{r_{m}+1} \int_{\pi /\left(b_{n}+1\right)}^{\pi} u^{\alpha-1} \sum_{j=0}^{\rho} p_{j, r_{m}-k} d u\right. \\
& \left.+\frac{\pi^{\alpha+1}}{(\alpha+1)\left(r_{m}+1\right)^{\alpha+1}} \int_{\pi /\left(b_{n}+1\right)}^{\pi} \frac{1}{u} \sum_{j=0}^{\rho} p_{j, r_{m}-k} d u\right\}
\end{aligned}
$$

In each integration, replace $u$ by $1 / w$ (remembering that $\rho=[1 / u]$ ) to get

$$
\begin{aligned}
I_{2}= & \frac{O(1)}{P_{n m}^{\beta \gamma}} \sum_{k=t_{m}+1}^{r_{m}}(k+1)\left\{\frac{1}{r_{m}+1} \sum_{l=0}^{b_{n}} \frac{1}{(l+1)^{\alpha+1}} \sum_{j=0}^{l} p_{j, r_{m}-k}\right. \\
& \left.+\frac{1}{\left(r_{m}+1\right)^{\alpha+1}} \sum_{l=0}^{b_{n}} \frac{1}{l+1} \sum_{j=0}^{l} p_{j, r_{m}-k}\right\} .
\end{aligned}
$$

The first sum on the right is equal to

$$
\begin{aligned}
A & =\frac{1}{\left(r_{m}+1\right) P_{n m}^{\beta \gamma}} \sum_{k=t_{m}+1}^{r_{m}}(k+1) \sum_{l=0}^{b_{n}} \frac{1}{(l+1)^{\alpha+1}} \sum_{j=0}^{l} p_{j, r_{m}-k} \\
& =\frac{1}{\left(r_{m}+1\right) P_{n m}^{\beta \gamma}} \sum_{l=0}^{b_{n}} \frac{1}{(l+1)^{\alpha+1}} \sum_{j=0}^{l} \sum_{k=t_{m}+1}^{r_{m}}(k+1) p_{j, r_{m}-k} .
\end{aligned}
$$

Using the following inequality

$$
\sum_{k=t_{m}+1}^{r_{m}}(k+1) p_{j, r_{m}-k} \leq \sum_{k=0}^{r_{m}} \sum_{s=0}^{r_{m}-k} p_{j s}
$$

we can write

$$
\begin{aligned}
A & =\frac{1}{\left(r_{m}+1\right) P_{n m}^{\beta \gamma}} \sum_{l=0}^{b_{n}} \frac{1}{(l+1)^{\alpha+1}} \sum_{k=0}^{r_{m}} \sum_{j=0}^{l} \sum_{s=0}^{r_{m}-k} p_{j s}=\frac{1}{\left(r_{m}+1\right) P_{n m}^{\beta \gamma}} \sum_{l=0}^{b_{n}} \sum_{k=0}^{r_{m}} \frac{P_{l, r_{m}-k}}{(l+1)^{\alpha+1}} \\
& =\frac{1}{\left(r_{m}+1\right) P_{n m}^{\beta \gamma}} \sum_{l=0}^{b_{n}} \sum_{k=0}^{r_{m}} \frac{P_{l k}}{(l+1)^{\alpha+1}} \leq \frac{1}{P_{n m}^{\beta \gamma}} \sum_{l=0}^{b_{n}} \sum_{k=0}^{r_{m}} \frac{P_{l k}}{(l+1)^{\alpha+1}\left(r_{k}+1\right)} .
\end{aligned}
$$

The second sum at the right hand side of (2.42) can be dominated in a similar manner:

$$
\frac{1}{\left(r_{m}+1\right)^{\alpha+1} P_{n m}^{\beta \gamma}} \sum_{k=0}^{r_{m}}(k+1) \sum_{l=0}^{b_{n}} \frac{1}{l+1} \sum_{j=0}^{l} p_{j, r_{m}-k} \leq \frac{1}{P_{n m}^{\beta \gamma}} \sum_{l=0}^{b_{n}} \sum_{k=0}^{r_{m}} \frac{P_{l k}}{(l+1)\left(r_{k}+1\right)^{\alpha+1}} \text {. }
$$

From (2.42)-(2.44) it follows that

$$
I_{2}=O\left(\Psi_{n m}\right)
$$

Similarly, by (2.21) 


$$
I_{3}=O\left(\Psi_{n m}\right)
$$

Using that (2.22),

$$
I_{4}=\frac{O(1)}{P_{n m}^{\beta \gamma}} \int_{\pi /\left(b_{n}+1\right)}^{\pi} \int_{\pi /\left(r_{m}+1\right)}^{\pi}\left(\frac{u^{\alpha}+v^{\alpha}}{u v}\right) P_{\rho \eta} d u d v
$$

We replace $y$ by $1 / y, v$ by $1 / w$, keeping in mind that $\rho=[1 / u]$ and $\eta=[1 / v]$. As a result, we obtain

$$
\begin{gathered}
I_{4}=\frac{O(1)}{P_{n m}^{\beta \gamma}} \int_{1 / \pi}^{\left(b_{n}+1\right) / \pi} \int_{1 / \pi}^{\left(r_{m}+1\right) / \pi}\left(\frac{1}{y^{\alpha+1} w}+\frac{1}{y w^{\alpha+1}}\right) P_{[y][w]} d y d w . \\
I_{4}=\frac{O(1)}{P_{n m}^{\beta \gamma}} \sum_{j=0}^{b_{n}} \sum_{k=0}^{r_{m}}\left(\frac{1}{(j+1)^{\alpha+1}(k+1)}+\frac{1}{(j+1)(k+1)^{\alpha+1}}\right) P_{j k}=O\left(\Psi_{n m}\right)
\end{gathered}
$$

Combining (2.33), (2.40), (2.45)-(2.47) results in (2.38).

\section{REFERENCES}

[1] Armitage D. H., Maddox I. J., A new type of Cesáro mean. Analysis 9 (1989) 195-206.

[2] Başarır M., Sonalcan O., On some double sequence spaces. J. Indiana cad. math. 21 (1999) 193200.

[3] Bromwich T. J., An introduction to the theory of infinite series. Macmillan and co. Ltd., New York (1965).

[4] Dağadur İ., Sezgek Ş. Deferred Cesàro mean and deferred statistical convergence of double sequences. J. İneq. And special func. 7-4 (2016) 118-136.

[5] Goffman C., Petersen G. M., Submethods of regular matrix summability methods. Canad. J. Math. 8 (1956) 40-46.

[6] Hamilton H. J., Transformations of double series. Bull. Amer. Math. Soc. 42 (1936) 275-283.

[7] Hardy G. H., On the convergence of certain multiple series. Proc. London math. Soc. 2-1 (1904) 124-128.

[8] Lal S., On the degree of approximation of functions belonging to the weighted $\left(L^{p}, \xi(t)\right)$-class by $(\mathrm{C}, 1)(\mathrm{E}, 1)$ means, Tamkang J. Math. 30-1(1999) 47-52.

[9] Lal S., On the degree of approximation of conjugate of a function belonging to the weighted $W\left(L^{p}, \xi(t)\right)$-class by matrix summability means of conjugate series of a fourier series, Tamkang J. Math, 31-4(2000) 279-288.

[10] McFadden L., Absolute Nörlund Summability, Duke Math. J. 9(1942) 168-207.

[11] Mittal M. L., Singh U., Mishra V. N., Approximation of signals (functions) belonging to the weighted $\left(L^{p}, \xi(t)\right)$-class by Nörlund means. Varahmihir J. Math. Sci. 6-1(2006) 383-392.

[12] Moore C. N., Summable series and convergence factors. Amer. Math. Soc. Colloq. Publ. 22 (1938).

[13] Móricz F., Rhoades B. E., Approximation by Nörlund means of double Fourier series for Lipschitz functions, J. of App. Theory 50 (1987) 341-358.

[14] Móricz F., Extension of the spaces $c$ and $c_{0}$ from single to double sequence. Acta math. Hungarica 57 (1991) 129-136.

[15] Osikiewicz J. A., Equivalence results for Cesàro submethods. Analysis 20 (2000) 35-43. 
[16] Patterson R. F., Analogues of some fundamental theorems of summability theory. Int. J. Math. Math. Sci. 23 (2000) 1-9.

[17] Qureshi K., On the degree of approximation of functions belonging to the weighted $\left(L^{p}, \xi(t)\right)$ class, Indian J. Pure Appl. Math. 13(1982) 471-475.

[18] Robison G. M., Divergent double sequences and series. Amer. Math. Soc. 28 (1926) 50-73.

[19] Tripathy B. C., Generalized difference paranormed statistically convergent sequences defined by Orlicz function in a locally convex spaces. Soochow J. Math. 30 (2004) 431-446.

[20] Ünver M., Inclusion results for four dimensional Cesàro submethods. Stud. Univ. Babeş Bolyai vmath. 58-1 (2013) 43-54.

[21] Yıldırım M., Karakuş F., On the degree of approximation to a function belonging to the weighted $\left(L^{p}, \xi(t)\right)$-class by $(N, p, q) E_{1}$ means, East Asian Mathematical J. 20(2004) 1-9.

[22] Yıldırım M., Karakuş F., The almost $(\mathrm{C}, 1)(\mathrm{E}, 1)$ summability of a fourier series and its conjugate series, Bull. Cal. Math. Soc. 98-4(2006) 285-294.

[23] Yıldırım M., Karakuş F., On $(N, p, q) C_{1}$ summability of the sequence $\left\{n B_{n}(x)\right\}$. E-Journal of New World Scienses Academy, 4-4(2009) 117-123.

[24] Zeltser M., On conservative and coercive SM-methods. Proc. Estonian acad. Sci. Phys. Math. 502 (2001) 76-85.

[25] Zeltser M., Investigation of double sequence spaces by soft and hard analytical methods. Diss. Math. Univ. Tartu. 25 Tartu Univ. Pres. Univ. of Tartu, faculty of mathematics and computer science, Tartu (2001).

[26] Zygmund A., Trigonometric series. J. Cambridge Univ. pres, Cambridge (1959). 